\title{
Minimum Number of Palettes in Edge Colorings
}

\author{
Mirko Horňák • Rafał Kalinowski • \\ Mariusz Meszka • Mariusz Woźniak
}

Received: 17 January 2012 / Revised: 30 January 2013 / Published online: 20 March 2013

(C) The Author(s) 2013. This article is published with open access at Springerlink.com

\begin{abstract}
A proper edge-coloring of a graph defines at each vertex the set of colors of its incident edges. This set is called the palette of the vertex. In this paper we are interested in the minimum number of palettes taken over all possible proper colorings of a graph.
\end{abstract}

Keywords Edge colouring · Palette of a vertex

\section{Mathematics Subject Classification 05C15}

The work of the first author was supported by Science and Technology Assistance Agency under the contract No. APVV-0023-10 and by Grant VEGA 1/0428/10. The research of the remaining three authors was partially supported by Polish Ministry of Science and Higher Education.

M. Horňák

Institute of Mathematics, P.J. Šafárik University, Jesenná 5, 04001 Košice, Slovakia e-mail: mirko.hornak@upjs.sk

R. Kalinowski $(\varangle) \cdot$ M. Meszka $\cdot$ M. Woźniak

Department of Discrete Mathematics, Faculty of Applied Mathematics AGH, Al. Mickiewicza 30, 30-059, Kraków, Poland

e-mail: kalinows@agh.edu.pl

M. Meszka

e-mail: meszka@agh.edu.pl

M. Woźniak

e-mail:mwozniak@agh.edu.pl 


\section{Introduction}

Let $G$ be a finite simple graph, let $C$ be a set of colors and let $f: E(G) \rightarrow C$ be an edge-coloring of $G$. We shall always assume that $f$ is proper, i.e., any two adjacent edges get distinct colors. The palette of a vertex $v \in V(G)$ with respect to $f$ is the set $S_{f}(v)$ of colors of edges incident to $v$.

Two vertices of $G$ are distinguished by a coloring $f$ if their palettes are distinct. There are many papers discussing colorings distinguishing vertices in a graph, that is, colorings with maximum possible number of pallettes (see, e.g., [1-3], and references given there). To our knowledge, this paper is the first one dealing with an opposite problem. Namely, we are interested in the minimum number of palettes taken over all possible proper (edge-) colorings of a graph. For a given graph $G$, we denote this number by $\check{s}(G)$ and call the palette index of $G$.

The minimum number of colors required in a proper coloring of a graph $G$ is called the chromatic index of $G$ and is denoted by $\chi^{\prime}(G)$. Recall that, by Vizing's theorem, the chromatic index of $G$ equals either $\Delta(G)$ or $\Delta(G)+1$. A graph with $\chi^{\prime}(G)=\Delta(G)$ is called class 1 , while a graph with $\chi^{\prime}(G)=\Delta(G)+1$ is called class 2 . Our first result on the palette index is (almost) obvious.

Proposition 1 The palette index of a graph $G$ is 1 if and only if $G$ is regular and class 1.

A proper coloring of $G$ using $\chi^{\prime}(G)$ colors is called minimum. In general, however, minimum colorings do not provide the minimum number of palettes. In our analysis the following lemma will be useful.

Lemma 2 If a graph $G$ is regular, then $\check{s}(G) \neq 2$.

Proof Suppose that $G=(V, E)$ is $r$-regular with $\breve{s}(G)=2$, and let $f$ be the corresponding coloring of $G$. Denote by $P_{1}$ and $P_{2}$ the palettes induced by $f$ and let $V_{i}=\left\{x \in V: S_{f}(x)=P_{i}\right\}, i=1,2$. Without loss of generality, we may suppose that $f$ is chosen in such a way that the set $P_{1} \backslash P_{2}$ is as small as possible. Since $P_{1} \neq P_{2}$ and $\left|P_{1}\right|=\left|P_{2}\right|$, there exists a color $\alpha \in P_{1} \backslash P_{2}$, as well as a color $\beta \in P_{2} \backslash P_{1}$. Clearly, the edges colored with $\alpha$ form a perfect matching of the subgraph $G\left[V_{1}\right]$ (induced in $G$ by the set $V_{1}$ ) and the same is true for the edges colored with $\beta$ and the subgraph $G\left[V_{2}\right]$. Since $\left\{V_{1}, V_{2}\right\}$ is a partition of $V$, by replacing $\alpha$ with $\beta$ we get a coloring that induces also two palettes $P_{1}^{\prime}, P_{2}^{\prime}$, but with $\left|P_{1}^{\prime} \backslash P_{2}^{\prime}\right|=\left|P_{1} \backslash P_{2}\right|-1$, a contradiction.

A set of edges $E^{\prime}$ of a graph $G$ with a coloring $f: E(G) \rightarrow C$ is called $f$-rainbow if $\left|f\left(E^{\prime}\right)\right|=\left|E^{\prime}\right|$, that is, if each edge of $E^{\prime}$ has different color. The following lemma deals with class 1 graphs $K_{2 k}$ and $K_{2 k+1,2 k+1}$.

Lemma 3 (a) For every minimum coloring $f$ of a complete graph $K_{2 k}$ with $k \neq 2$, there exists an $f$-rainbow perfect matching in $K_{2 k}$.

(b) There exists a minimum coloring $f$ of $K_{2 k+1,2 k+1}$ such that there is an $f$-rainbow perfect matching in $K_{2 k+1,2 k+1}$.

Proof The part (a) follows, for instance, from results given in [4]. 
To prove the part (b), assume that $V\left(K_{2 k+1,2 k+1}\right)=X \cup Y$, where $X=\left\{x_{0}, x_{1}\right.$, $\left.\ldots x_{2 k}\right\}$ and $Y=\left\{y_{0}, y_{1}, \ldots y_{2 k}\right\}$. In order to construct an appropriate coloring $f$, color the edges $x_{i+j} y_{i+2 j}, j=0,1, \ldots 2 k$ (where indices are taken modulo $2 k+1$ ), with the color $i$. An $f$-rainbow perfect matching consists of the edges $x_{i} y_{i}, i=0,1, \ldots 2 k$.

\section{Complete Graphs}

Since the graphs $K_{1}$ and $K_{2 k}$ are class 1 , we have $\check{s}\left(K_{1}\right)=\check{s}\left(K_{2 k}\right)=1$. On the other hand, it is easy to see that the minimum coloring of $K_{n}$ for odd $n$ induces $n$ distinct palettes. Indeed, each palette has $n-1$ colors. That means that at each vertex exactly one color is missing. Further, since $n$ is odd, each color misses at least one vertex. Consequently, each color misses exactly one vertex and missing colors are distinct for distinct vertices. However, by increasing the number of colors we can reach the number of palettes 3 or 4 .

The aim of this section is to determine palette indices of complete graphs:

\section{Theorem 4}

$$
\check{s}\left(K_{n}\right)=\left\{\begin{array}{lll}
1, \text { if } n \equiv 0 & (\bmod 2) \quad \text { or } n=1 \\
3, \text { if } n \equiv 3 & (\bmod 4) & \\
4, \text { if } n \equiv 1 & (\bmod 4) &
\end{array}\right.
$$

The proof follows from five partial results, namely Proposition 1, Proposition 5, Theorem 6, Theorem 7 and Proposition 8.

\subsection{Complete Graphs with Palette Index 3}

First, we deal with the case of orders in congruence class 3 modulo 4.

Proposition 5 If $k \geq 0$, then $\check{s}\left(K_{4 k+3}\right)=3$.

Proof We begin with showing the existence of a coloring $f$ inducing three palettes. Partition the vertex set $V$ of the graph $K_{4 k+3}$ into three sets: two sets $X$ and $Y$, each having $2 k+1$ elements, and one one-element set, say $\{u\}$.

We first color the edges of the complete graph induced by $X$ using $2 k+1$ colors from the set $A=\left\{a_{1}, a_{2}, \ldots, a_{2 k+1}\right\}$ to obtain a minimum coloring of $K_{2 k+1}$. Thus, there is exactly one color of $A$ missing at each vertex of $X$.

Next, we color the edges of the complete graph induced by $Y$ using $2 k+1$ colors from the set $B=\left\{b_{1}, b_{2}, \ldots, b_{2 k+1}\right\}$ to obtain a minimum coloring of $K_{2 k+1}$. Thus, there is exactly one color of $B$ missing at each vertex of $Y$. We assume that $A$ and $B$ are disjoint.

Now, we color each edge $u x$ joining the vertex $u$ to a vertex $x \in X$ in such a way that $u x$ gets the color from the set $A$ missing at $x$. Analogously, we color each edge $u y$ joining $u$ to a vertex $y \in Y$ in such a way that $u y$ gets the color from the set $B$ missing at $y$. Therefore, in this moment, the (partial) palettes of vertices of $X$ coincide with the set $A$ and those of vertices of $Y$ coincide with $B$. 
Finally, we color the edges between $X$ and $Y$ using the colors from a set $C$ with $|C|=2 k+1$ and $C \cap(A \cup B)=\varnothing$, in order to get a minimum coloring of the bipartite graph $K_{2 k+1,2 k+1}$.

Then all vertices of $X$ have the palette $A \cup C$, all vertices of $Y$ have the palette $B \cup C$ and the vertex $u$ has the palette $A \cup B$.

The above defined coloring shows that $\breve{s}\left(K_{4 k+3}\right) \leq 3$. However, by Lemma 2 , $\check{s}\left(K_{4 k+3}\right) \neq 2$, and, by Proposition $1, \breve{s}\left(K_{4 k+3}\right) \neq 1$.

The next theorem provides a full characterization of complete graphs with the palette index 3 .

Theorem 6 The palette index of the complete graph $K_{n}$ equals 3 if and only if $n \equiv 3$ $(\bmod 4)$.

Proof Suppose that $\breve{s}\left(K_{n}\right)=3$. By Proposition 1 then $K_{n}$ is class 2 , so that $n$ is odd and $n \geq 3$. There is a coloring of $K_{n}$ inducing three distinct palettes $P_{i}, i=1,2,3$. Let $V_{i}$ be the set of all vertices of $K_{n}$ with palette $P_{i}$ and let $n_{i}=\left|V_{i}\right|, i=1,2,3$. Clearly, we have $\left|P_{i}\right|=n-1$ and $\left|P_{i} \backslash P_{j}\right|=\left|P_{j} \backslash P_{i}\right|, i, j=1,2,3$.

Observe first, that there is no color belonging to all three palettes. Indeed, otherwise this color would induce a perfect matching of $G$, which is impossible ( $n$ is odd). Consequently, a color of any edge joining $V_{i}$ and $V_{j}, i \neq j$, induces a perfect matching of the graph $G\left[V_{i} \cup V_{j}\right]$. Therefore, $n_{i}+n_{j}$ with $i \neq j$ is always even. So, all $n_{i}$ 's are of the same parity, and, since $n=n_{1}+n_{2}+n_{3}$ is odd, all $n_{i}$ 's are odd. This implies in particular, that there is no color belonging to exactly one palette. Hence, each color belongs to exactly two palettes.

Set $\xi=\left|P_{1} \backslash P_{2}\right|$ and $\eta=\left|P_{1} \cap P_{2}\right|$. Thus, $\xi+\eta=n-1$. Moreover, since $P_{3}=\left(P_{1} \backslash P_{2}\right) \cup\left(P_{2} \backslash P_{1}\right)$ and $\left|P_{2} \backslash P_{1}\right|=\left|P_{1} \backslash P_{2}\right|=\xi$, we have $2 \xi=n-1$ and, as a consequence, we obtain $\xi=\eta=\frac{n-1}{2}$. Of course, by symmetry it holds

$$
\left|P_{i} \backslash P_{j}\right|=\left|P_{i} \cap P_{j}\right|=\frac{n-1}{2} \quad \text { for } \quad i, j=1,2,3, i \neq j .
$$

Let $E_{i, j}$ denote the set of all edges joining $V_{i}$ and $V_{j}$. Since all $n_{i}$ 's are odd, $\left|E_{i, j}\right|$ is odd, too. Moreover, for every color $\alpha$ from $P_{i} \cap P_{j}$, the number $e_{\alpha}$ of edges in $E_{i, j}$ colored with $\alpha$ is odd (otherwise, $n_{i}-e_{\alpha}$ is odd and then color $\alpha$ is missing at some vertex in $V_{i}$ ). Thus the total number of colors in $P_{i} \cap P_{j}$ is odd, what implies $\frac{n-1}{2} \equiv 1$ $(\bmod 2)$, and finally $n \equiv 3(\bmod 4)$.

The reverse implication has been proved as Proposition 5.

\subsection{Complete Graphs $K_{4 k+5}$ with $k \neq 1$}

Theorem 7 If $n=4 k+5, k \neq 1$, then $\check{s}\left(K_{n}\right)=4$.

Proof By Proposition 1, Lemma 2 and Theorem 6 we see that $\check{s}\left(K_{n}\right) \geq 4$. Therefore, it will be sufficient to find a coloring of $K_{n}$ inducing four palettes.

Partition the vertex set $V$ of the graph $K_{n}$ with $n=4 k+5$ into four sets: two sets $X$ and $Y$, each having $2 k+1$ elements, one one-element set, say $\{u\}$ and one two-element set, say $\{v, w\}$. Let $X=\left\{x_{0}, x_{1}, \ldots, x_{2 k}\right\}$ and $Y=\left\{y_{0}, y_{1}, \ldots, y_{2 k}\right\}$. 


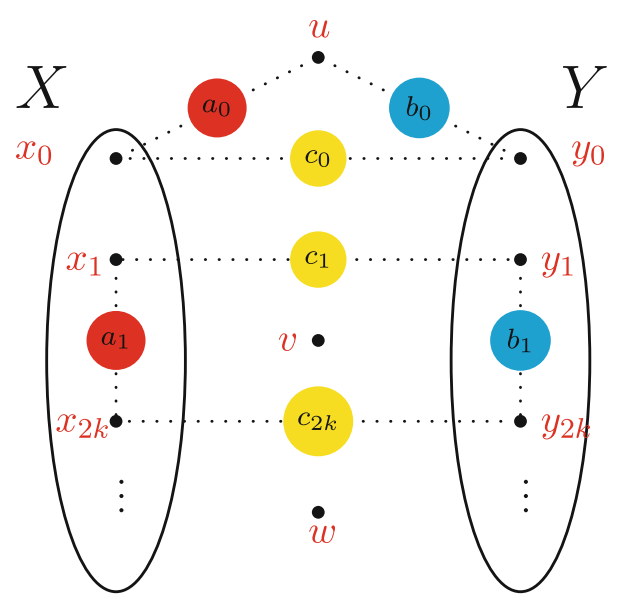

Fig. 1 After step 1

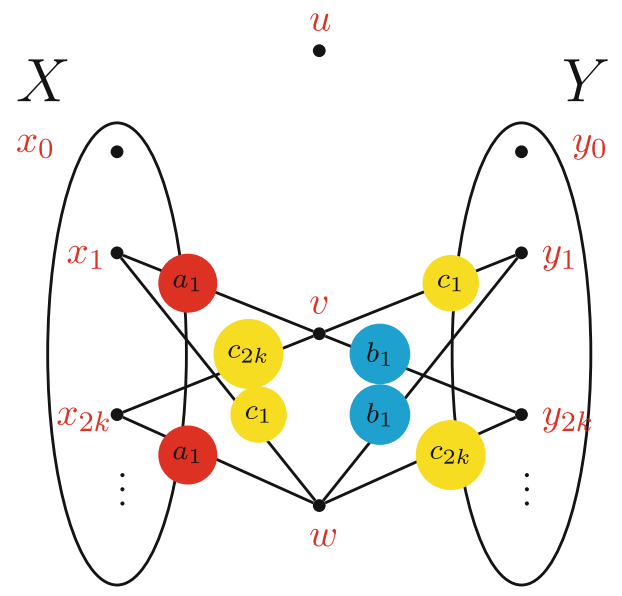

Fig. 2 After step 2

Step 1. Let $A=\left\{a_{0}, a_{1}, \ldots, a_{2 k}\right\}, B=\left\{b_{0}, b_{1}, \ldots, b_{2 k}\right\}$ and $C=\left\{c_{0}, c_{1}, \ldots, c_{2 k}\right\}$ be three pairwise disjoint sets of $2 k+1$ colors. Consider (arbitrary) minimum colorings $f_{A}: E\left(K_{n}[X \cup\{u\}]\right) \rightarrow A$ and $f_{B}: E\left(K_{n}[Y \cup\{u\}]\right) \rightarrow B$. By Lemma 3 a) there exists an $f_{A}$-rainbow perfect matching $M_{A}$ in the graph induced by $X \cup\{u\}$ and an $f_{B}$-rainbow perfect matching $M_{B}$ in the graph induced by $Y \cup\{u\}$. If $H$ is the subgraph of $K_{n}$ isomorphic to $K_{2 k+1,2 k+1}$ with bipartition $\{X, Y\}$, by Lemma 3 b) there is a minimum coloring $f_{C}: E(H) \rightarrow C$ such that $H$ has an $f_{C}$-rainbow perfect matching $M_{C}$.

Without loss of generality we may assume that $M_{A}$ consists of $k+1$ edges $u x_{0}$, $x_{1} x_{2 k}, \ldots, x_{k} x_{k+1}$ colored with colors $a_{0}, a_{1}, \ldots, a_{k}$, respectively; $M_{B}$ consists of $k+1$ edges $u y_{0}, y_{1} y_{2 k}, \ldots, y_{k} y_{k+1}$ colored with colors $b_{0}, b_{1}, \ldots, b_{k}$, respectively; and $M_{C}$ consists of $2 k+1$ edges $x_{i} y_{i}, i=0,1, \ldots, 2 k$ colored with colors $c_{0}, c_{1}, \ldots, c_{k}$, respectively.

We uncolor the edges belonging to $M_{A} \cup M_{B} \cup M_{C}$ (see Fig. 1). 


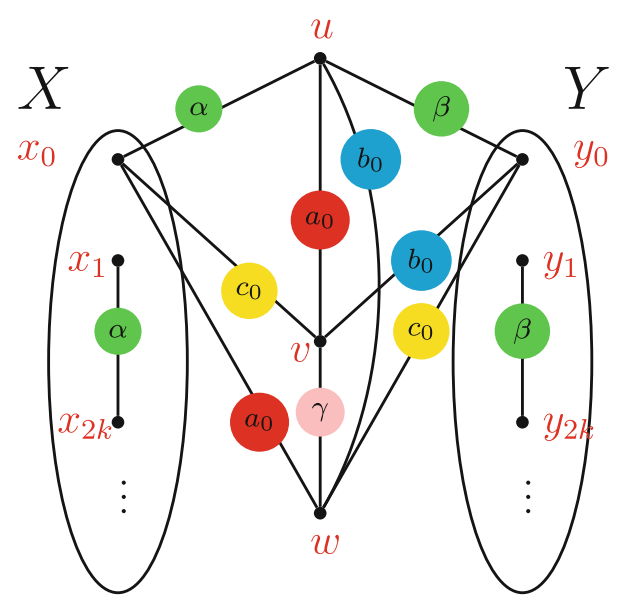

Fig. 3 After step 3. For the sake of clarity of the drawing edges $x_{i} y_{i}$, colored with $\gamma$, are not presented

Step 2. For each $i, 1 \leq i \leq k$ we color the four edges joining the vertices $v, w$ to the vertices $x_{i}, x_{2 k-i+1}$, as well the four edges joining the vertices $v, w$ to the vertices $y_{i}, y_{2 k-i+1}$ in the following way (see Fig. 2):

- the edges $v x_{i}$ and $w x_{2 k-i+1}$ are colored with $a_{i}$, the color missing at both vertices $x_{i}$ and $x_{2 k-i+1}$;

- the edges $w y_{i}$ and $v y_{2 k-i+1}$ are colored with $b_{i}$, the color missing at both vertices $y_{i}$ and $y_{2 k-i+1}$;

- the edges $w x_{i}$ and $v y_{i}$ are colored with $c_{i}$, the color missing at both vertices $x_{i}$ and $y_{i}$

- the edges $v x_{2 k-i+1}$ and $w y_{2 k-i+1}$ are colored with $c_{2 k-i+1}$, the color missing at both vertices $x_{2 k-i+1}$ and $y_{2 k-i+1}$;

Observe that after this step all vertices of $X$ except for $x_{0}$ have the (partial) palette $A \cup C$, while all vertices of $Y$ except for $y_{0}$ have the palette $B \cup C$.

Step 3. In this step we color all the edges containing the vertices $v$ and $w$ which were not colored yet, as well as the edges uncolored in Step 1.

First, the edges $v u$ and $w x_{0}$ are colored with $a_{0}$, the edges $w u$ and $v y_{0}$ are colored with $b_{0}$, and the edges $v x_{0}$ and $w y_{0}$ are colored with $c_{0}$.

Next, we pick three distinct colors $\alpha, \beta, \gamma$ so that $\{\alpha, \beta, \gamma\} \cap(A \cup B \cup C)=\emptyset$ and color the edges of $M_{A}$ with $\alpha$, the edges of $M_{B}$ with $\beta$ and the edges of $M_{C}$ with $\gamma$. Finally, we color the last edge not colored yet, $v w$, with $\gamma$.

Observe that the vertices of $X$ have the palette $A \cup C \cup\{\alpha, \gamma\}$, the vertices of $Y$ have the palette $B \cup C \cup\{\beta, \gamma\}$, and both vertices $v$ and $w$ have the palette $\left\{a_{i}, b_{i}\right.$ : $i=0,1, \ldots, k\} \cup C \cup\{\gamma\}$. The fourth palette, that of the vertex $u$, is $A \cup B \cup\{\alpha, \beta\}$.

This finishes the proof of the theorem. 


\subsection{The Complete Graph $K_{9}$}

To complete the proof of Theorem 4, it is enough to settle the palette index of $K_{9}$.

Proposition $8 \check{s}\left(K_{9}\right)=4$.

Proof For the same reason as in the Sect. 2.2, it suffices to define a coloring $f$ of edges of $K_{9}$ inducing four palettes.

Suppose that $V\left(K_{9}\right)=\left\{x_{i}: i=1,2, \ldots, 9\right\}$ and let $f$ be a coloring of $K_{9}$ with 13 colors, described by the following symmetric $9 \times 9$ matrix $A=\left(a_{i j}\right)$, namely by setting $f\left(x_{i} x_{j}\right)=a_{i j}, i, j=1,2, \ldots, 9, i \neq j$ (the empty diagonal corresponds to the fact that in $K_{9}$ there are no loops):

$$
A=\left(\begin{array}{lllllllll} 
& 1 & 2 & 3 & 4 & \overline{0} & 6 & \overline{1} & 5 \\
1 & & 3 & 2 & 6 & 4 & \overline{0} & 5 & \overline{1} \\
2 & 3 & & 1 & 5 & \overline{1} & 4 & 6 & \overline{0} \\
3 & 2 & 1 & & \overline{2} & 9 & 7 & \overline{0} & 8 \\
4 & 6 & 5 & \overline{2} & & 8 & \overline{1} & 9 & 7 \\
\overline{0} & 4 & \overline{1} & 9 & 8 & & 5 & 7 & 6 \\
6 & \overline{0} & 4 & 7 & \overline{1} & 5 & & 8 & 9 \\
\overline{1} & 5 & 6 & \overline{0} & 9 & 7 & 8 & & 4 \\
5 & \overline{1} & \overline{0} & 8 & 7 & 6 & 9 & 4 &
\end{array}\right)
$$

Clearly, the elements of the $i$-th row (as well as those of the $i$-th column) of the matrix $A$ form the palette of the vertex $x_{i}$ with respect to $f$. Hence, the palettes are: $\{1,2,3,4,5,6, \overline{0}, \overline{1}\}$ for the vertices $x_{1}, x_{2}, x_{3},\{1,2,3,7,8,9, \overline{0}, \overline{2}\}$ for the vertex $x_{4},\{4,5,6,7,8,9, \overline{1}, \overline{2}\}$ for the vertex $x_{5}$, and $\{4,5,6,7,8,9, \overline{0}, \overline{1}\}$ for the vertices $x_{6}, x_{7}, x_{8}, x_{9}$.

\section{Cubic Graphs}

Theorem 9 Let $G$ be a connected cubic graph.

If $G$ is class 1 , then $\check{s}(G)=1$.

If $G$ is class 2 and has a perfect matching, then $\breve{s}(G)=3$.

If $G$ is class 2 without a perfect matching, then $\check{s}(G)=4$.

Proof The first statement follows from Proposition 1.

Suppose now that $G$ is class 2 and has a perfect matching $M$. Then the graph $G-M$ is the union of disjoint cycles with at least one of an odd length. Each minimum coloring of this graph induces exactly three palettes (partial in $G$ ). Observe that by coloring the edges of $M$ with a new color each palette acquires the new color, hence the number of palettes is three as before and $\breve{s}(G) \leq 3$. On the other hand, $\breve{s}(G) \geq 3$ by Proposition 1 and Lemma 2, so we are done.

Suppose finally that $G$ is class 2 , but does not contain a perfect matching. Observe that, since all palettes have three elements, each minimum coloring of $G$ (which uses four colors) induces at most four palettes. For the same reason as above we have $\breve{s}(G) \geq 3$. Thus, it suffices to show that it is impossible to have three palettes. 
So, assume there exists a coloring $f$ of $G$ inducing three palettes $P_{1}, P_{2}$ and $P_{3}$. Denote by $V_{1}, V_{2}$ and $V_{3}$ the corresponding sets of vertices of $G$. Observe first that there is no color belonging to all three palettes (otherwise $G$ would have a perfect matching).

Since $G$ is connected, we may suppose without loss of generality that there are edges joining $V_{1}$ to $V_{2}$, and, consequently, $P_{1} \cap P_{2} \neq \emptyset$. For any color $a \in P_{1} \cap P_{2}$ the edges colored with $a$ form a perfect matching $M_{a}$ of the graph $G\left[V_{1} \cup V_{2}\right]$. This implies, in particular, that there is no color belonging just to $P_{3}$. Indeed, suppose that $b \in P_{3} \backslash\left(P_{1} \cup P_{2}\right)$. Then the edges colored with $b$ form a perfect matching $M_{b}$ of the graph $G\left[V_{3}\right]$ and $M_{a} \cup M_{b}$ is a perfect matching of $G$, a contradiction. Thus, $P_{3}=\left(P_{1} \backslash P_{2}\right) \cup\left(P_{2} \backslash P_{1}\right)$.

Recall that in a cubic graph all palettes are of size three. Therefore, $\left|P_{1} \backslash P_{2}\right|=$ $\left|P_{2} \backslash P_{1}\right|$ and $\left|P_{3}\right|=\left|\left(P_{1} \backslash P_{2}\right) \cup\left(P_{2} \backslash P_{1}\right)\right| \equiv 0$ (mod 2$)$, a contradiction.

Acknowledgments An important part of this work was done during the 15th C5 Graph Theory Workshop, Rathen 2011. The authors are very grateful to Ingo Schiermeyer and all his group of organizers of that meeting.

Open Access This article is distributed under the terms of the Creative Commons Attribution License which permits any use, distribution, and reproduction in any medium, provided the original author(s) and the source are credited.

\section{References}

1. Burris, A.C., Schelp, R.H.: Vertex-distinguishing proper edge-colourings. J. Graph Theory 26, 73-82 (1997)

2. Černý, J., Horňák, M., Soták, R.: Observability of a graph. Math. Slovaca 46, 21-31 (1996)

3. Edwards, K., Horňák, M., Woźniak, M.: On the neighbour-distinguishing index of a graph. Graph Combin. 22, 341-350 (2006)

4. Woolbright, D.E., Fu, H-L.: On the existence of rainbows in 1-factorizat-ions of $K_{2 n}$. J. Combin. Des 6, 1-20 (1998) 